\title{
7 Corpora that Talk to Each Other
}

The idea that lies at the basis of a data-driven history of philosophy is to enable researchers and readers to delve into XML formatted corpora that are both human-readable and machine-readable. History of philosophy is particularly apt for multilingual semantic alignment experiments because of its essential, non-redundant lexicon. The need and added value of providing easy access to complex, highly structured philosophical content through corpora that talk to each other have been highlighted in the literature. However, they have not yet been fulfilled (Pozzo 2016). Given that concepts do not have an independent life and are mediated by linguistic expressions and only from these expressions do they have their specific meaning, a thorough lexicographical investigation is "indispensable and unavoidable for determining both the conceptual apparatus and the philosophical problems" (Sgarbi 2012, ix-x).

In this chapter, I shall start with a user-based analysis of existing repositories in alphabets and hanzi 汉字 characters. An effective approach is to develop add-on modules and plug-ins for already existing open-source tools. The objective is to assess the potential of available repositories and the coherence among existing research e-platforms and e-infrastructures through a comparative analysis of currently operating formats while considering international standards of learning content to ensure their export both directly and through metadata harvesting procedures.

\subsection{Multilingual Corpora}

A text corpus is a large and unstructured set of texts that once were on paper but today are electronically stored and processed. Text corpora are used to carry out statistical analysis and hypothesis testing, check occurrences and validate linguistic rules within a specific language territory. Originally drawn out manually, corpora are now automatically derived from source texts. Online corpora with query engines are a part of today's life. Think of clusters with multiple pieces of speech-tagged corpora, each using a different set of tags and corpus-query language (Baker 1993).

A corpus may contain texts in a single language (monolingual corpus) or textual datasets in multiple languages (multilingual corpora). Multilingual corpora that have been specially formatted for side-by-side comparison are called aligned parallel corpora. To make corpora more useful for linguistic research, they are annotated, i.e., enriched with further information. One of the most dynamic proj- 
ects in the construction of parallel text corpora of modern languages and the development of reliable tools for alignment and morphosyntactic annotation of words is InterCorp (Bozzi 2015, 37). ${ }^{85}$

At the Leibniz Institute for the German Language in Mannheim, the program area Corpus Linguistics is dedicated to developing and testing tools for the automatic analysis of corpora and the construction and application of quantitative mathematical models of explorative corpus analysis. Researchers in Mannheim are working on preparation and annotation of corpora, analytically based metrization of their properties and relations of linguistic units, extraction, reconstruction, and exploration of linguistic knowledge from corpora of texts in natural languages, fostering applications in the field of text analysis and text technology, and supporting linguistic theory formation. ${ }^{86}$

Computational concept modeling is a process that exploits a successful synthesis of working practices in the humanities and computational linguistics. Once humanities scholars have agreed to study a corpus, they first identify appropriate levels and categories of analysis, they then perform annotations on a subsample of the corpus that acts as reference data, which become the basis for "machine learning experiments with candidate model classes, including additional tools or data resources" (Kuhn 2020, 76). The token class of a sequence of characters cannot be determined before the semantic analysis has been carried through, as names and variable names are lexically identical but constitute different token classes (Li Wenchao 2015). It is the reader who calls the semantic analyzer (say, the root of a Greek-Latin concept pair such as phantasia/imaginatio) and checks if the sequence requires lemmatization. In this case, information has to flow back not merely from the text itself, but from the semantic analyzer back to the reader, which poses a challenge to the design: "Whenever we are tempted to talk about the hermeneutic meaning of a text, we should talk instead of the meaning of the text for someone, that is, the meaning of a work" (Bevir 1999, 74).

As a matter of fact, in the lexica of non-roman languages, a copious introduction of Greek and Latin forms has taken place, the consequence of the diffusion in Europe of a set of scientific lexica, which were in relevant part globally shared. Researchers understand and elaborate texts in different languages with the option of interrogating them across alphabets from the standpoint of a meta-language to be chosen by the user. They rely on transcriptions, translations, and distillations of texts (e.g., Yahya 2014; Yi Zhuang 2014). A data-driven

85 https://ucnk.ff.cuni.cz/cs/, visited 6 May 2021.

86 http://www1.ids-mannheim.de/kl.html, visited on 6 May 2021. 
history of philosophy shall consider texts how they have been transmitted and used within the individual cultural communities, which today happens through websites, for example, in the Islamic Philosophy Online portal. ${ }^{87}$

Researchers in history of philosophy ought to have the gift of multilingualism because a new sociolinguistics of multilingualism is now being forged: one that takes account of the new communicative order and the particular conditions of our times while retaining a central concern with the "processes involved in the construction of social differences and social inequalities" (Gardner and Martin-Jones 2012, 1). As a matter of fact, it is easy to see that one groundbreaking aspect of history of philosophy lies in its addressing multilingual aspects. On one side, we have texts and terms; on the other ideas and problems. The solution is an innovative way of working with the history of scientific lexica within cultural studies to the advantage of twenty-first-century philosophers.

For example, annotating Kant's work as a corpus is an endeavor that has been increasingly carried out over more than sixty years alongside the progress of computational linguistics. The start was given by the Allgemeiner Kantindex (Martin 1967; Roser and Mohrs 1992), which gave Kant's words in non-inflected form and is currently preserved within the Korpora.org platform. ${ }^{88}$ A giant leap forward was achieved by Tullio Gregory (1967) and Norbert Hinske (1982), respectively with the Lessico Intellettuale Europeo (now on TEI) and the KantIndex (built on TUSTEP), which granted access to Kant's writings in lemmatized form with metadata and semantic annotations that are interoperable also as regards multilingualism (i.e., Kant's use of Greek, Latin, German, and French terms). The next giant leap forward is expected to be achieved by recontextualizing Kant within multilingual philosophical corpora around computational concept modeling, making it possible to kick-start the so-called computational history of ideas (Betti and Van den Berg 2016).

Generally, one can take up the text-corpus method that derives a set of abstract rules that govern a natural language from texts in that language and explores how it relates to others. The scientific approach is empirical, but in different ways: to digitalize and operationalize Kant's work in two languages presupposes philosophical expertise to control the translation.

The nine volumes of Kant's printed works, with their 1,580,00 words, offer material for a full lemmatization and a formidable basis for reflected text analytics. Starting from an Urtext of German lemmata, it is possible to create an induced network of concepts based on which to pursue empirically verifiable hy-

87 https://muslimphilosophy.org, visited on 6 May 2021.

88 https://korpora.zim.uni-duisburg-essen.de/kant/, visited on 6 May 2021. 
potheses on meaning shifts over the centuries. At the Center for Reflected Text Analytics of the University of Stuttgart, ${ }^{89}$ the interdisciplinary research team led by Jonas Kuhn has developed methods for theory- and data-driven corpus analysis (Kuhn 2020). These methods enable scholars to formulate hypotheses regarding systematic patterns in distributing specific concepts in a corpus and test them empirically. For example, one might try to verify a presumed tendency for a school of thinking to translate the term A as A' in the context of debate $\mathrm{X}$, but as A" in other contexts, which is what happened with the first translation in French of Kant's Kritik der reinen Vernunft, when the word Vernunft was rendered with raison in some contexts and with entendement in others (Müller and Pozzo 1988).

The corpora alignment of the German Urtext with its Chinese translation (see below chapter 8) might eventually be carried out on the Kant Online platform. The platform is currently under construction. ${ }^{90}$ Kant Online has the Kant-Lexikon (Willaschek at al. 2015) as its nomenclature. To name an analogous endeavor, one can have a look at the Nietzsche Online platform (Nietzsche 2011), which provides access to the complete edition of Friedrich Nietzsche's works and letters by Giorgio Colli and Mazzino Montinari, as well as to almost all publications published by De Gruyter on Nietzsche's works and reception. In addition to about seventy volumes of the Nietzsche edition, the platform offers access to monographs and reference works such as the Nietzsche-Wörterbuch (Van Tongeren et al. 2004) and the issues of the Nietzsche-Studien-all in all, more than 110,000 book pages. However, Nietzsche Online offers significantly more than the sum of its printed content. Below each text are witnesses presented both as images and transcriptions (this is especially important for manuscripts). Searches can be made to obtain links between individual sentences or specific terms to corresponding sentences in other text witnesses (if any) and, if necessary, to their presence within the reconstructed object and the corresponding stemma codicum. The content of the texts in the database is extensively linked so that secondary literature dedicated to the passage by Nietzsche that is being read can be accessed directly. The reconstructed text is enriched by a philological apparatus that justifies critical choices between variants and historicalcritical explanations that provide information about the content and context of the work (Pozzo 2014).

89 https://www.creta.uni-stuttgart.de, visited on 6 May 2021.

90 https://www.degruyter.com, visited on 6 May 2021. 


\subsection{Digital Libraries}

It is agreed that a digital library is a space in which to bring together collections, services, and people for creating data, providing access and preserving data, and consequently for information and knowledge. The first occurrence of the syntagma dates back to 1971. The first digital library was Project Gutenberg, initiated by Michael Hart to create a library of freely reproducible electronic versions of printed books. ${ }^{91}$ Nevertheless, digital libraries existed well before the internet. They were up and running long before they were called that way, namely in the decades when the only available supports were punch cards, then replaced by magnetic tapes and later by floppy disks. Among the first to develop computational systems for literary text was the National Research Council of Italy (CNR), starting from the reform of its statutes in 1963 when its range of action finally expanded to 360 degrees with the foundation of SSH dedicated centers of studies. CNR made possible enterprises such as the Index Thomisticus by Roberto Busa, which was conceived as early as 1946 and eventually published in 1980 in fiftysix volumes with the support of the Institute of Computational Linguistics (ILC) directed by Antonio Zampolli. ${ }^{92}$ Think also of the Lessico giuridico italiano project that Luigi Lombardi Vallauri created at the Institute of Theory and Technique of Legal Information (ITTIG), which has been active since $1968 .^{93}$

\subsubsection{Institute for the European Intellectual Lexicon and History of Ideas}

For scope, impact, and longevity, however, the priority belongs to the Institute for the European Intellectual Lexicon and History of Ideas (ILIESI), founded by Tullio Gregory with the collaboration of Tullio De Mauro in 1964 at the Institute of Philosophy of Sapienza University as a center of studies of CNR. Over the decades, the ILIESI has been a place of study and training for young researchers, most of whom today have become eminent scholars. His raison d'être was to make scholars a digital library available, enabling a more rigorous historical reading of the philosophical texts and documents of the period in which European cultural and scientific terminology was formed (Liburdi 2000, 2007).

The ILIESI digital library (Lessico Intellettuale Europeo: Banca dati di testi filosofici dell'età moderna) initially consisted of 100 philosophical and scientific

91 https://www.gutenberg.org, visited on 6 May 2021.

92 http://www.ilc.cnr.it, visited on 6 May 2021.

93 http://www.ittig.cnr.it, visited on 6 May 2021. 
texts published between 1600 and 1800, including many by Galileo, Descartes, Spinoza, Leibniz, Vico, and Kant. ${ }^{94}$ These were lemmatized texts, the metadata of which made easy access possible and, consequently, annotation to find lemmata and phrases, expanding the search from one text to others, with the researcher becoming an agent and the document a dynamic one, given that access was required by different users at different times.

Against the speculative approach of idealistic historiography, which left the detailed study of the text in the background, and with a good dose of CNR trademarked innovation, Gregory opened the season of a history of ideas closely linked to the history of terminology based on the assumption that ideas do not live in a hyper-uranium world, pure and immaculate. Instead, ideas are embodied in linguistic, impure, often ambiguous signs. Linguistic signs are carriers of a long history, a crossroads of multiple experiences in the intertwining of diverse currents of thought and different languages in the continuous transcription and translation from one culture to another (Gregory et al. 1967-2021). In consideration of Eugenio Garin's claim that history of philosophy implies shedding light on the function of philosophers in the history of a civilization (Ciliberto 2011), one might argue that the approach set forward by Gregory was perhaps more about rethinking and bringing to completion the long tradition of historicism started by Vico, rather than about opposing and destroying its idealistic forms.

For these reasons, the ILIESI focuses on the phenomenon of cultural migration, which accompanies the whole history of civilizations while involving continuous relations and reciprocal exchanges among diverse cultures. ILIESI researchers investigate several epochs under the assumption that at the root of the history of philosophy and the sciences and more generally of the history of ideas lie textual corpora developed in the context of each discipline over the centuries. Historical semantic tools consider technical uses and ambiguities, synchronic and diachronic interrelations, translations, and transpositions across lexical fields. The specificity of the methodology requires keeping close to texts, individual terms, and lexical families. Hence, the publication of lexica, indices and concordances, the setting up of databases, in which data processing is applied to technical terminology in the humanities (Gregory et al. 1967-2021).

94 http://www.iliesi.cnr.it, visited on 6 May 2021. One renowned example for all: Paolo Galluzzi's (1979) ground-breaking monograph on the notion of momentum in Galileo. 


\subsubsection{World Digital Library}

While the ILIESI digital library contains metadata-rich and Europeana formatted editions of about 500 texts, which are highly representative of philosophical and scientific thought in Greek, Latin, French, Italian, English, German, and Spanish, the World Digital Library (WDL) offers a much larger array of texts, actual books, manuscripts, maps, photographs, and other primary materials in one-hundredseventy languages. ${ }^{95}$ To date, the WDL is one of the most exciting examples of corpora that talk to each other, for it provides its users with navigation tools and content descriptions in seven languages-the six official languages of the United Nations, namely Arabic, Chinese, English, French, Russian, and Spanish, with the addition of Portuguese. The WDL was launched by the Librarian of Congress, James H. Billington, before the US National Commission for UNESCO in 2005. After some meetings dedicated to prototype development, the library has been operative on the internet since 2009, promoting intercultural dialogue, increasing the volume and the variety of cultural content offered on the internet, providing resources to educators, scientists, and the public at large, while developing cooperation agreements among national libraries and partner institutions all over the world to diminish the digital divide between poor and rich countries. These objectives require creating and managing modular research teams through the organizational structures provided by technology and digital communications and techniques for cooperation with other SSH, STEM, and ICT disciplines.

\subsubsection{Twenty-four European Languages}

Recent research has focused on corpora and resources for high resource languages with particular attention to the twenty-four official languages of the European Union-Bulgarian, Croatian, Czech, Danish, Dutch, English, Estonian, Finnish, French, German, Greek, Hungarian, Irish, Italian, Latvian, Lithuanian, Maltese, Polish, Portuguese, Romanian, Slovak, Slovene, Spanish, Swedish-that are all represented in Europeana ${ }^{96}$ and European Cultural Heritage Online (ECHO). ${ }^{97}$ Both work towards multilingualism: Europeana with metadata in the twentyfour official languages to support cultural heritage in its digital transformation,

95 www.wdl.org, visited on 6 May 2021.

96 www.europeana.eu, visited on 6 May 2021.

97 https://echo.mpiwg-berlin.mpg.de/home, visited on 6 May 2021. 
while ECHO has created an infrastructure to bring cultural heritage on the internet.

\subsubsection{Greek and Latin}

Greek and Latin are preserved in long-standing, relatively small thesauri that make their lexical analysis quite useful. Hence, we rely today on several successful endeavors such as the Index Thomisticum Treebank, ${ }^{98}$ Computational Historical Semantics, ${ }^{99}$ the Thesaurus Linguae Graecae: A Digital Library of Greek Literature, ${ }^{100}$ the Thesaurus Linguae Latinae Online, ${ }^{101}$ the Library of Latin Texts, ${ }^{102}$ the Patrologia Latina, ${ }^{103}$ the Late Latin Charter Treebank, ${ }^{104}$ the Archivio della Latinità Italiana Medievale, ${ }^{105}$ and, most importantly, their connection on the Linguistic Linked Open Data Cloud. ${ }^{106}$

\subsubsection{Arabic}

Looking into the Islamic world, philosophers rely on the Islamic Philosophy Online portal. ${ }^{107}$ For instance, Islamic philosophy insists on God having infinite names and attributes, the most important one being the Mother of Names (Umm al-Asma، الأسماء أمّاء). The names themselves are considered in the following order: living (al-Havy الحيّ), knowing (al-'Alim العليم), willing (al-Murīd المريد), powerful (al-Qādir قادر), speaking (al-Mutakallim المتكلمّ), hearing (al-Sami، السمعى), seeing (al-Basir البصير). All names presuppose the category of living (al-Havy الحيّ (الحيّ), which has priority over all and fulfills precisely the same function of the Aristotelian substance (ousía oúoí $\alpha$ ) (Yahya and Sahli 2014). Also, we might include opinion (zann ظنّ) aقين (Smirnov 2018, 11-12).

98 https://itreebank.marginalia.it, visited on 6 May 2021.

99 https://www.comphistsem.org/home.html, visited on 6 May 2021.

$100 \mathrm{http}: / /$ stephanus.tlg.uci.edu/, visited on 6 May 2021.

$101 \mathrm{https} / / /$ www.thesaurus.badw.de/tll-digital/tll-open-access.html, visited on 6 May 2021.

$102 \mathrm{http}: / /$ www.brepols.net, visited on 6 May 2021.

$103 \mathrm{http} / / /$ patristica.net/latina, visited on 6 May 2021.

104 https://zenodo.org/record/1197357\#.X34RxS8QOMw, visited on 6 May 2021.

105 http://alim.unisi.it, visited on 6 May 2021.

$106 \mathrm{https} / / /$ linguistic-lod.org/llod-cloud, visited on 6 May 2021.

107 https://muslimphilosophy.org, visited on 6 May 2021. 


\subsubsection{Chinese}

The Chinese counterpart of the ILIESI is the Key Concepts of Chinese Thought and Culture project, whose raison d'être is to select concepts in Chinese thought and culture that reflect the characteristics of the country's traditional culture and the Chinese people's way of thinking while embodying the core values of China (Wang Lin and Han Zhen 2015-2020). ${ }^{108}$ One should also mention the Chinese Text Project. ${ }^{109}$

\subsubsection{Global Perspective}

In chapter 8, I will look into the conditions of possibility of translating Kant in twenty-first-century academic and cultural contexts (Schlüter and Hohenegger 2020). The question is how to figure out ways to insert old and new philosophical translations into text corpora, i.e., into metadata-rich and fully interoperable sources, translations, bibliographies, indexes, lexica, and encyclopedias. For example, the World Humanities Forum series is an interesting result of the convergence between SSH and state-of-the-art technology (Choi 2014, 423-428). ${ }^{110}$ The following years will see a joint effort to mutually align texts from alphabets and characters, involving not only European languages and Chinese but also Arabic, Farsi, Hebrew, Korean, Japanese, Neo-Greek, Russian, Turkish, and Sanskrit. Again, insisting on the centrality of text requires today seeing beyond paper supports into the realm of big data, which means into text corpora.

\subsection{CLARIN, DARIAH, OPERAS}

Historians of philosophy should take up the habit of reusing and conferring datasets and tools from and to three infrastructures that belong to the strategy working group on Social and Cultural Innovation of the ESFRI, namely the Common Language Resources and Technology Infrastructure (CLARIN), ${ }^{111}$ which serves the community of computational linguistics while granting access to substantial resources on historical corpora translation issues, the Digital Research

108 https://www.chinesethought.cn, visited on 6 May 2021.

109 https://ctext.org/, visited on 6 May 2021.

$110 \mathrm{http}: / /$ worldhumanitiesforum.com/eng/main/, visited on 6 May 2021.

111 https://www.clarin.eu, visited on 6 May 2021. 
Infrastructures for the Arts and Humanities (DARIAH), ${ }^{112}$ which serves the community of the digital humanities and arts, and the European Research Infrastructure for the Development of Open Scholarly Communication in the Social Sciences and Humanities (OPERAS). ${ }^{113}$

According to a generic but effective definition, research infrastructures are complex and varied instruments that allow users from the scientific community to share resources, tools, and data products. First and foremost, the centers of these three research infrastructures provide services to ensure the long-term preservation of the data and software, including newly created data and tools. To make this work, each infrastructure imposes specific requirements on the description (metadata) of the resources, the formats of the resources (to achieve syntactic interoperability), and provisions for specifying the meaning of elements in the resource (to ensure semantic interoperability). Second, as regards creating and describing resources, each infrastructure provides various tools to assist researchers in meeting these requirements.

Just as astronomers need a virtual observatory to study the stars and other distant objects in our galaxy and beyond, researchers in the humanities need digital infrastructures to access information and knowledge embedded in digital content. Most importantly, researchers are welcome to contribute to the lifespan of the infrastructure with new research projects that might either yield new data and software or enrich existing data with new annotation layers. New data and software should be made available to the research community for a variety of reasons. These include ensuring transparency, verification enablers, and possibly the replication of research results. Most research outcomes are funded with public money and should therefore be made available to the whole research community. They should be easily accessible also after the research project has ended. In this way, other researchers can benefit from them in their research, and the original researchers can benefit from reusing them later.

\subsubsection{Common Language Resources and Technology Infrastructure}

In 2012, CLARIN was established in the form of a European Research Infrastructure Consortium (ERIC) and took up the mission to create and maintain an infrastructure to support the sharing, use, and sustainability of language data and research tools in the SSH. With centers located in twenty-one European member

112 https://www.dariah.eu, visited on 6 May 2021.

113 https://operas.hypotheses.org, visited on 6 May 2021. 
states, CLARIN provides easy access to digital language data (in written, spoken, or multimodal form) for scholars in the SSH and beyond (Odjik 2016). CLARIN also offers advanced tools to discover, explore, exploit, annotate, analyze, and combine linguistic datasets wherever they are located. This is enabled through a networked federation of centers, including language data repositories, service centers, and knowledge centers, all of them predisposed with single sign-on access for members of the academic community in the participating countries. Tools and data from different centers are interoperable. Data collections can be combined, and tools from various sources can be chained to perform complex operations to support researchers in their work.

Finding digital data relevant to humanities research requires consulting text corpora with rich linguistic annotations, lexica, lexical databases, audio recordings (possibly with annotation), audio-visual data for language documentation, micro-comparative databases, typological databases, and many others. Services to apply the software to the digital data in a user-friendly manner are a core functionality offered by CLARIN. Most of the data and many of the software tools have already existed before. However, they require downloading and installing software packages, which often happen only on specific computer platforms and depend on other software packages. The data and the software have been produced interoperable. In several cases, dedicated new services and interfaces must be created that did not exist before. This lowers the barrier for using the data and software significantly to benefit linguistic research on a much larger scale than ever before. CLARIN resources embrace a pool of web applications that offer multiple interfaces to the same data. Each interface has its target in terms of the complexity of the query and the user's expected technical proficiency. Dedicated interfaces usually restrict usage, but an environment is created to avoid this by combining different interfaces in one application. It is challenging to create a correct query from scratch, but making minor adaptations to an existing well-formed question is much more comfortable. ${ }^{114}$

\subsubsection{Digital Research Infrastructures for the Arts and Humanities}

DARIAH is a network of people, expertise, information, knowledge, content, methods, tools, and technologies from nineteen European member states. DARIAH brings together individual state-of-the-art digital arts and humanities activities and scales their results to a European level. It preserves, provides access to,

114 https://www.clarin.eu, visited on 6 May 2021. 
and disseminates research and research outputs that stem from these collaborations and ensures that best practices, methodological and technical standards are followed. In 2014, DARIAH was established as an ERIC to empower researchers with digital methods to create, connect and share knowledge about culture and society, to build tools, knowledge, and groups to facilitate the digital transformation of the arts and humanities, and to champion technology in the service of the arts and humanities. DARIAH integrates digital arts and humanities research and activities from across Europe, enabling transnational and transdisciplinary approaches through its competence centers. It promotes the further development of research methods in the arts and humanities, documenting state of the art, supporting the reuse of research data, focusing on particular challenges including diversity, provenance, multimedia collections, and granularity, while acting as a coordinator and integrator for communities of practice. In fact, as I have suggested above in chapter 6, DARIAH is particularly interesting for understanding how cultural innovation can happen with the input of its twenty-one working groups. ${ }^{115}$

All things considered, DARIAH impacts a series of interconnected domains: education, science, and culture in the first instance, but also society, policy, and the digital economy. While building services for researchers working with ICTbased methods, DARIAH ensures the long-term accessibility of their work, thus directly contributing to understanding the cultural, economic, social, and political life in Europe and beyond. Finally, DARIAH offers teaching material and teaching opportunities to develop digital research skills (Romary et al. 2016; Edmond et al. 2020). ${ }^{116}$

\subsubsection{European Research Infrastructure for the Development of Open Scholarly Communication in the Social Sciences and Humanities}

OPERAS provides tools and systems for improving the quality and speed of the peer review process, which has become today a critical success factor for the future of scholarly communication. The issue is especially important for the academic books sector, where quality assessment processes need to be made more transparent, perhaps via systems for open peer review and streamlined to serve the research community better. Although not yet established as an ERIC (it will be in 2025), OPERAS implements several online collaborative

115 https://www.dariah.eu/activities/working-groups-list/, visited on 6 May 2021. 116 https://www.dariah.eu, visited on 6 May 2021. 
tools for authoring from Open Source and proprietary providers. Finally, it develops a high number of publishing systems and an existing trend for more opensource development. At the same time, OPERAS aims at integrating with an everincreasing set of third-party tools, enhancements, and discoverability services based on the general recommendation to simplify the distribution process through a service tool that receives a feed of data and files and automatically distributes them to all the appropriate locations for a determinate publisher or journal. ${ }^{117}$

\subsection{Forward Look}

CLARIN, DARIAH, and OPERAS provide a perspective that sheds light on how a $5 \mathrm{G}$ supported context of reading might look like in 2028. However, we are not yet in 2028, and we would rather stick to what we have at hand. One thing is sure: history of philosophy needs critical editions and, together with them, a methodology of text interpretation. Since these presuppositions are not always established in non-Western areas, it should be a task of the Committee on History of Philosophy of the FISP to offer validated contacts to arrange a knowledge transfer of the methods from experienced editors and interpreters to those who might need it. ${ }^{118}$

The basic idea is that the study of corpora with their shifts in meaning and hybridizations is the most effective tool for cultural history and for studying languages in the plasticity of their history, which has a considerable effect on the work of translators as well. An interesting experiment might be setting up and running a set of pyramidal e-books on philosophical contents, say, following Robert Darnton's (2009) depiction of a literary genre that does not exist right now. The reader shall start at the top level by perusing the highest, most uncomplicated, and most general narrative. From there on, s/he shall follow the links and go into deeper levels of multimedia content, according to his/her needs and interests. The most uncomplicated narratives (the top of the pyramid) shall be organized, starting from a few key concepts. I am talking about an approach for presenting linear texts and multimedia content that takes up the challenge of renewing scientific rigor and opening up new formats for multimedia publishing and broadcasting. It is a vast territory that is up to be explored, described, and mapped out.

117 https://operas.hypotheses.org, visited on 6 May 2021.

118 https://www.fisp.org/committees, visited on 6 May 2021. 
Let me make an example. The terms representation and imitation are indeed translations for the same term, namely mimesis (mímēsis $\mu^{\prime} \mu \eta$ бıৎ) (Pozzo 2010). However, they have different meanings according to the contexts-e.g., following either Plato on ideas or Aristotle on tragedy or Dante on interpretation:

\begin{abstract}
Let us assume a user is working his/her way on paideía $\pi \alpha \mathrm{s} \delta \varepsilon^{\prime} \alpha$. The following issue might attract him/her: "education is the imitation of role models." Role models are to be found in the family, in the community, and in society as a whole. By browsing the highest narrative of an e-book dedicated to paideía, the user encounters very soon a reference to Aristotle's

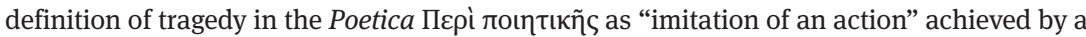
noble character. Step 1: Movie-material (e.g., scenes from Saving Private Ryan by Steven Spielberg); Step 2: RaiEducational Docufilm on Aristotle's Poetica; Step 3: Aristotle, Poetica 1449 b 24-28; Step 4: 1542 Renaissance Latin Translation by Alessandro Paccio; Step 5: 1570 Renaissance Italian Translation by Ludovico Castelvetro; Step 6: 1980 Contemporary French Translation (Aristotle 1980) by Dupont-Roc and Lallot; Step 7: 1997 Contemporary English Translation by Stephen Halliwell; Step 8: 2007 Contemporary Italian Translation by Domenico Pesce; Step 9: Historical and Contemporary Translations in Other Languages; Step 10:

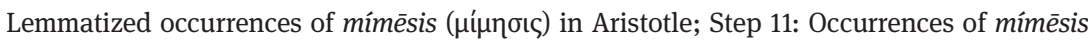
in ancient authors such as Plato, Demosthenes, Cicero, etc.; Step 12: Encyclopedia articles; Step 13: Journal articles; Step 14: Monographs; Step 15: Position of a philosophical argument: "Et que se passe-t-il quand on rend mímēsis par représentation au lieu d'imitation?" demanded Barbara Cassin (2004, s.v.). This was the decision taken by Roselyne Dupont-Roc and Jean Lallot: in order to point out the ambivalence, they came to the conclusion that 'mimeisthai' ought to be translated into French by 'représenter' instead by 'imiter.' In fact, mimeisthai: "sauf présence d'éléments discriminants dans le contexte, 'représenter

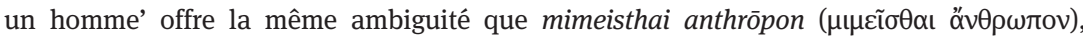
alors que la traduction traditionnelle par 'imiter' sélectionne abusivement l'interprétation de l'accusatif comme celui du modèle." The reason Dupont-Roc and Lallot provide is: "les connotations théâtrales de ce verbe et surtout la possibilité de lui donner pour complément, comme à mimeisthai, indifférement l'objet 'modèle' et l'objet 'produit'-au lieu qu'imiter excluait ce dernier, le plus important-ne pouvaient qu'emporter la décision” (Aristotle 1980, 20).
\end{abstract}

One hardly needs to point out how many issues of transdisciplinarity and multiculturalism historians of philosophy are confronted with (Taylor 1994; Scarantino 2013). Philosophical texts constitute the common cultural foundation that lies at the origin of pluralism from antiquity to our days. At stake is the ability to come to terms with ambiguities to acquire a diversity of both internal and external origin productively. In this context, history of philosophy facilitates creative thinking. It provides students and scholars with methods and content for both plastic and differentiated expression and enriching logical arguments using metaphors and iconic references. Disciplinary boundaries can always be questioned for the sake of a more encompassing perspective. 
In this chapter, I have laid out some use cases of corpora, corpus linguistics, computational linguistics, natural language processing, and their contribution to digital humanities. In connection with the CLARIN resource families, the use cases implement FAIR data standards, which stimulate the reuse and repurposing of available research data, thereby enabling scholars in the SSH to increase their productivity and open new research venues in and across disciplines that address one or more of the multiple societal roles of language. Language is the carrier of cultural content and information, "one of the central components of the identity of individual groups, cultures, or nations, as an instrument for human expression, as an object for study and preservation” (ESFRI 2018, 213). In conclusion, this chapter is about igniting research agendas that "illustrate the added value of well-supported access to the wealth of data types that are available for multiple languages ... the research initiatives for the study of migration patterns, intellectual history, language variation across period and region, dynamics in mental health conditions, customer opinions and parliamentary discourse, just to name a few" (De Jong 2019, 123). 